\title{
POSICIÓN Y CONDUCTA TRIETÁPICA BCG DE LAS TOP10 COOPERATIVAS AGROALIMENTARIAS ESPAÑOLAS, EN EL PERIODO 2015-2018
}

\section{Antonio Colom Gorgues *a, Rosa M. Florensa Guiu ${ }^{\mathrm{b}}$, Pilar Cos Sánchez ${ }^{\mathrm{c}}$}

a) Escuela Técnica Superior de Ingeniería Agraria, Departamento de Administración de Empresas, Universidad de Lleida; email: antonio_colom@hotmail.com ; ORCID: 0000-0001-7129-2539

$\left.{ }^{b}\right)$ Escuela Superior Politécnica, Departamento de Administración de Empresas, Universidad de Lleida; email: rosa.florensa@udl.cat ; ORCID:0000-0002-1791-3266

c) Facultad de Derecho, Economía y Turismo, Departamento de Administración de Empresas, Universidad de Lleida; email: pilar.cos@udl.cat ; ORCID: 0000-0001-7882-6847

\section{RESUMEN:}

Con esta comunicación se quiere resumir la investigación sobre Cooperativismo Agroalimentario a nivel de España, aplicando el modelo del Boston Consulting Group (BCG), para analizar el posicionamiento y conducta del conjunto TOP10 por nivel de facturación de las cooperativas agroalimentarias españolas, en las tres etapas de los años 2016, 2017 y 2018.

En España el cooperativismo agroalimentario está avanzando para subirse al tren de la integración, incremento de dimensión y aumento del poder de negociación, como elementos esenciales asociados al buen resultado empresarial cooperativo. Para ello, y en paralelo, se están investigando y poniendo en práctica distintos modelos de crecimiento, de reestructuración socioeconómica y de modelo de gobierno interno cooperativo. Se ha analizado la posición y conducta de las 10 Cooperativas Agroalimentarias que más facturan en España, viendo sus diferencias estructurales, de Cuota Relativa de Mercado y de Crecimiento, en las tres campañas de 2016, 2017 y 2018, a través de los resultados del citado análisis BCG. El itinerario seguido por el cooperativismo agroalimentario español se ve en el camino de esfuerzo en superar sus limitaciones y retos, y adaptación de su modelo organizativo para ir mejorando en su desempeño productivo, comercial, social y en relación con el respeto medioambiental.

Palabras Clave: Cooperativismo Agroalimentario en España, Modelo del Boston Consulting Group, Cuota Relativa de Mercado, Crecimiento de la Cuota Relativa de Mercado.

\section{INTRODUCCIÓN Y OBJETIVO}

El cooperativismo agroalimentario español, siguiendo el modelo mediterráneo de atomización cooperativa, está muy desarrollado en casi todas las Comunidades Autónomas. La distribución geográfica por CC.AA. de las 3.190 Cooperativas Agroalimentarias españolas se muestra en el cuadro 1 y gráfica 1.

Cuadro 1 y Gráfica 1. Distribución geográfica por CC.AA. en 2018 de las Cooperativas Agroalimentarias españolas. Número Total: 3.190 cooperativas agroalimentarias

\begin{tabular}{|c|l|c|c|c|}
\hline & Comunidadades Autónomas & $\begin{array}{c}\mathbf{N}^{\circ} \\
\text { Cooperativas }\end{array}$ & $\%$ & $\begin{array}{c}\% \\
\text { Acumulado }\end{array}$ \\
\hline $\mathbf{1}$ & ANDALUCÍA & 713 & $22,35 \%$ & $\mathbf{2 2 , 3 5 \%}$ \\
\hline $\mathbf{2}$ & CASTILLA LA MANCHA & 431 & $13,51 \%$ & $\mathbf{3 5 , 8 6 \%}$ \\
\hline $\mathbf{3}$ & CASTILLA Y LEÓN & 337 & $10,56 \%$ & $\mathbf{4 6 , 4 3} \%$ \\
\hline $\mathbf{4}$ & COMUNIDAD VALENCIANA & 333 & $10,44 \%$ & $\mathbf{5 6 , 8 7 \%}$ \\
\hline $\mathbf{5}$ & CATALUÑA & 293 & $9,18 \%$ & $\mathbf{6 6 , 0 5 \%}$ \\
\hline $\mathbf{6}$ & EXTREMADURA & 278 & $8,71 \%$ & $\mathbf{7 4 , 7 6 \%}$ \\
\hline $\mathbf{7}$ & ARAGÓN & 191 & $5,99 \%$ & $80,75 \%$ \\
\hline $\mathbf{8}$ & GALICIA & 165 & $5,17 \%$ & $85,92 \%$ \\
\hline $\mathbf{9}$ & REGIÓN MURCIA & 122 & $3,82 \%$ & $89,75 \%$ \\
\hline $\mathbf{1 0}$ & NAVARRA & 87 & $2,73 \%$ & $92,48 \%$ \\
\hline $\mathbf{1 1}$ & PAíS VASCO & 67 & $2,10 \%$ & $94,58 \%$ \\
\hline $\mathbf{1 2}$ & CANARIAS & 59 & $1,85 \%$ & $96,43 \%$ \\
\hline $\mathbf{1 3}$ & LA RIOJA & 45 & $1,41 \%$ & $97,84 \%$ \\
\hline $\mathbf{1 4}$ & ISLAS BALEARES & 31 & $0,97 \%$ & $98,81 \%$ \\
\hline $\mathbf{1 5}$ & MADRID & 19 & $0,60 \%$ & $99,40 \%$ \\
\hline $\mathbf{1 6}$ & PRINCIPADO ASTURIAS & 14 & $0,44 \%$ & $99,84 \%$ \\
\hline $\mathbf{1 7}$ & CANTABRIA & 5 & $0,16 \%$ & $100,00 \%$ \\
\hline & TOTALES $\ldots$ & $\mathbf{3 . 1 9 0}$ & $\mathbf{1 0 0} \%$ & \\
\hline
\end{tabular}

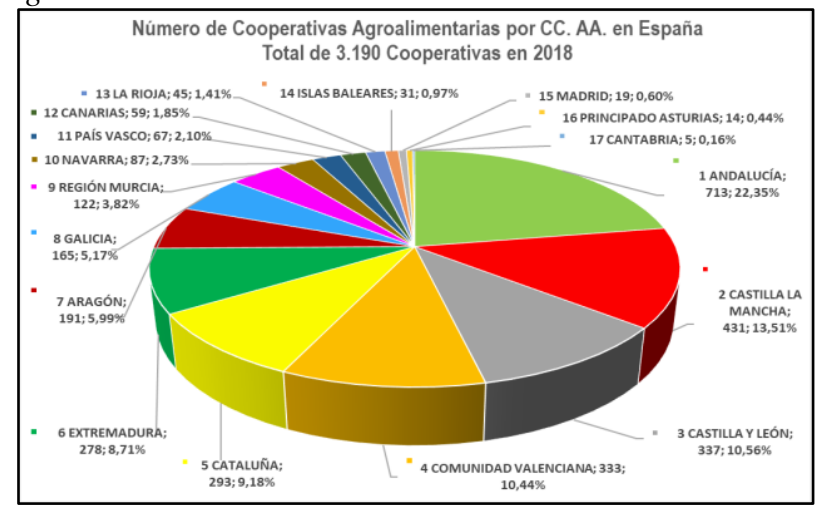

Fuente: Elaboración propia a partir de OSCAE (2020)

Como TOP 6 en número de cooperativas agroalimentarias por CC.AA. destacan: en primer lugar Andalucía como la CC.AA. con mayor número de cooperativas agroalimentarias: 713 (22,35\%), en segundo lugar Castilla La Mancha con 431 (13,51\%), en tercer lugar Castilla y León con 337 (10,56\%), en cuarto la Comunidad Valenciana con $333(10,44 \%)$, en quinto Cataluña con $293(9,18 \%)$, y en sexto lugar Extremadura con 278 (8,71\%). Estas TOP 6 Comunidades Autónomas integran casi el 75\% (74,76\%) del número de cooperativas agroalimentarias españolas, o sea, existe gran concentración. La Comunidad de Aragón sigue en el séptimo lugar con 191 cooperativas (5,99\%), en octavo está Galicia con 165 (5,17\%), en noveno la Región de Murcia con 122 (3,82\%), en décimo está Navarra con 87 (2,73\%), en undécimo el País Vasco con 67 (2,10\%), en undécimo Canarias con 59 (1,85\%) y en duodécimo está La Rioja con 45 $(1,41 \%)$. Las demás Comunidades Autónomas tienen un número de cooperativas que supone < $1 \%$ : así se anota como decimotercera la Comunidad de Islas Baleares con 31 cooperativas (0,97\%), como 
decimocuarta Madrid con 19 (0,60\%), como decimoquinta el Principado de Asturias con $14(0,44 \%)$ y finalmente como decimosexta está Cantabria con $5(0,16 \%)$.

Cuando nos referimos al peso económico de las cooperativas medido por su facturación, que da una estimación de su dimensión económica, se producen algunos cambios de orden si tomamos como referencia la lista anterior de la tabla1. Ver la distribución de la facturación cooperativa por CC.AA. en el cuadro 2 y la gráfica 2. En efecto, si nos fijamos en las cooperativas TOP 6 como en el caso del número, se mantiene Andalucía como CC.AA. también líder según la facturación de las cooperativas ubicadas en dicha región con unos 11.258 M€ (38\%), pero en segundo lugar aparece la Comunidad Valenciana con $2.812 \mathrm{M} €(9,5 \%)$, en tercer lugar Castilla La Mancha con $2.753 \mathrm{M} €(9,3 \%)$, en cuarto lugar Cataluña con $2.071 \mathrm{M} €$ (7\%), en quinto lugar Castilla y León con $2.041 \mathrm{M} €(6,9 \%)$ y en sexto Extremadura con $2.015 \mathrm{M} €(6,8 \%)$.

Cuadro 2 y Gráfica 2. Facturación de las Cooperativas Agroalimentarias españolas por CC. AA. En el año 2018. Total Facturación en 2018: 29.635 Millones de Euros

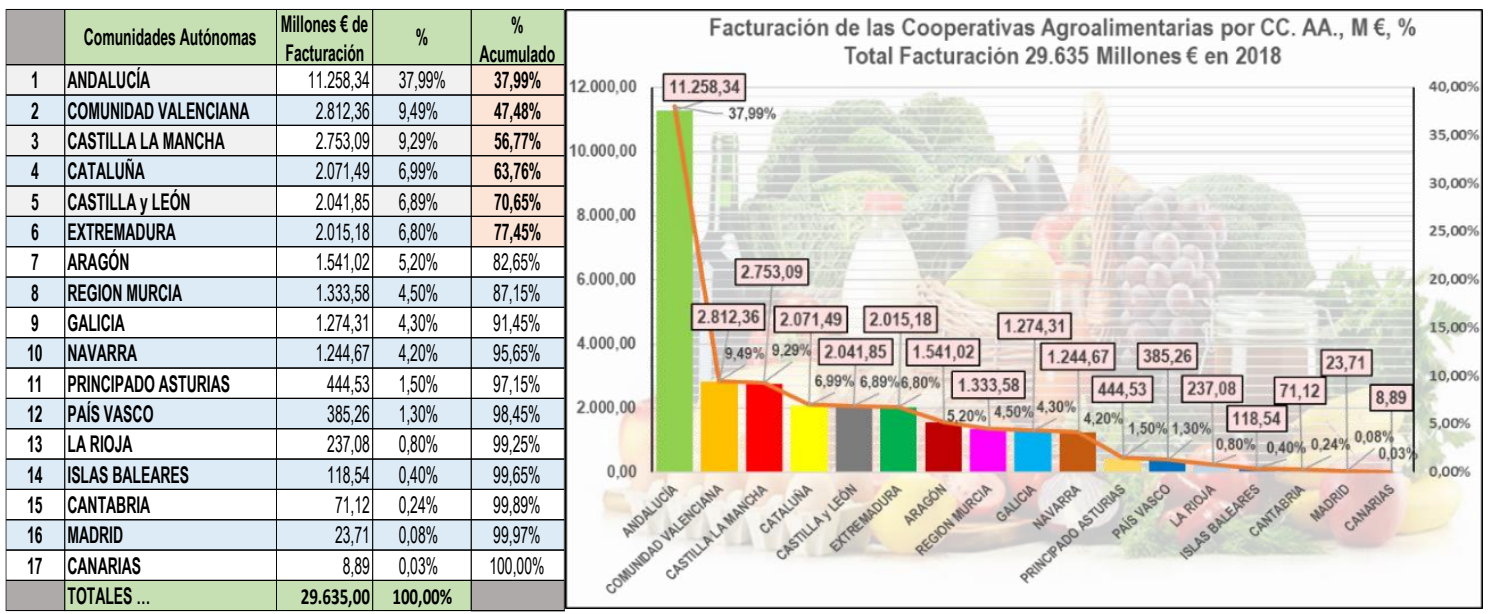

Fuente: Elaboración propia a partir de OSCAE (2020)

El Objetivo de esta comunicación es presentar la dinámica de comportamiento y posición de las cooperativas agroalimentarias con mayor dimensión y nivel de facturación de España, aplicando el modelo del Boston Consulting Group (BCG).

\section{ESQUEMA METODOLÓGICO}

La Matriz Portafolio del Boston Consulting Group (BCG) de Henderson (1973), es una herramienta para el análisis estratégico empresarial (ver la gráfica 3 ), analizando las dos variables siguientes:

- La Cuota Relativa de mercado de la sociedad cooperativa, para expresar su posición competitiva en el mercado, en un año o campaña del año n. Esta variable está relacionada con la capacidad de la empresa de generar Cash-Flow.

Gráfica 3. Esquema del modelo BCG. Fuente: Elaboración propia, de Colom, Cos y Florensa (2019)

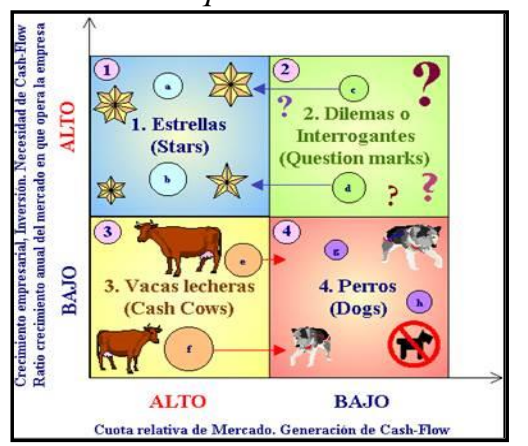

La Tasa de Crecimiento en el mercado para la sociedad cooperativa, desde la campaña anterior a la actual ([cuota campaña del año $n$ - cuota campaña del año $n-1]$ / cuota campaña del año $n-$ 1). Esta otra variable induce a la posibilidad de necesitar o aplicar Cash-Flow (para inversión, I+D+T+i, crecimiento empresarial).

El "\% de Cuota Relativa de mercado" se coloca en el eje horizontal y el "\% de Tasa de Crecimiento en el mercado" en el eje vertical de los ejes coordenados. Ambos ejes se dividen en dos zonas ALTA y BAJA cuya frontera es la media de ambas variables respectivas. Se forma una cuadrícula de 4 loci (ver la figura 3):

- ALTA-ALTO: "Estrellas": Alta cuota relativa de mercado y alto \% tasa de crecimiento en el mercado. Se trata de empresas con una muy buena posición que les dará opción a generar buenos niveles de Cash-Flow y posibilidades para inversión, innovación y crecimiento.

- BAJA-ALTO: "Interrogantes": Baja cuota relativa de mercado y alto \% de tasa de crecimiento en el mercado. Se trata de un posicionamiento que da lugar a un interrogante (?). La empresa tiene cuota relativa de mercado baja y en cambio tiene gran crecimiento en cuota de mercado desde la campaña anterior.

- ALTA-BAJO: "Vacas Lecheras": Alta cuota relativa de mercado, pero bajo \% de tasa de crecimiento. Ello posiciona en una condición, aprovechando su estatus de alta cuota relativa para generar alto nivel de cash-flow, pero en cambio olvida o las circunstancias se lo impiden, el crecimiento (falta de inversión e innovación, problemas de demanda, problemas estructurales, etc.). 
- BAJA-BAJO: "Perros": Baja cuota relativa de mercado y bajo \% de tasa de crecimiento de la cuota. Estas características llevan a clasificar estas empresas como las peor posicionadas, tal vez con una dinámica negativa de pérdida de peso y de competitividad, que pueden abocarlas a una situación de deterioro. No obstante, también tienen cabida aquí, empresas de pequeña dimensión que tienen una cuota relativa de mercado pequeña, adecuada a su índole local o regional, atienden a un nicho o pequeño segmento de mercado, y no se plantean ninguna estrategia de crecimiento.

\section{ANÁLISIS DINÁMICO BCG DE LAS TOP10 COOPERATIVAS AGROALIMENTARIAS ESPAÑOLAS EN LOS AÑOS 2016-2018. RESULTADOS, ANÁLISIS Y DIAGNÓSTICO}

En este apartado analizamos el grupo TOP10 de cooperativas agroalimentarias españolas, siguiendo el esquema y metodología del Boston Consulting Group de Henderson. Los datos se han obtenido de Cooperativas Agroalimentarias de España, a través de la publicación del OSCAE (2018, 2019, 2020). En el cuadro 3 y la gráfica 4 se muestran los datos y los resultados de posicionamiento 2016-2017-2018.

Cuadro 3. Datos y Posicionamiento BCG 2016-2018 de las TOP10 Coop. Agroalimentarias de España

\begin{tabular}{|c|c|c|c|c|c|c|c|c|c|c|c|c|c|c|c|c|c|c|c|}
\hline $\mathrm{N}^{0}$ & Cooperativa & CC. AA. & Grado & $\mid \begin{array}{c}\mathrm{N}^{\circ} \\
\text { Socios }\end{array}$ & Empleados & \begin{tabular}{|c|} 
Cifra de \\
Ventas \\
2011 \\
(M/€) \\
\end{tabular} & \begin{tabular}{|c|} 
Cifra de \\
Ventas \\
2016 \\
(M/G) \\
\end{tabular} & $\begin{array}{c}\text { \%Cuota } \\
\text { Relativa } \\
\text { TOP10 } \\
\text { 2016 }\end{array}$ & 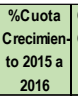 & \begin{tabular}{|l} 
Clase \\
Coop \\
BCG \\
2016 \\
\end{tabular} & \begin{tabular}{|c|} 
Cifra de \\
Ventas \\
2017 \\
(M/€) \\
\end{tabular} & \begin{tabular}{|c|}
$\%$ Cuota \\
Relativa \\
TOP10 \\
2017 \\
\end{tabular} & \begin{tabular}{|c|}
$\%$ Cuota \\
Crecimien- \\
to 2016 a \\
2017
\end{tabular} & $\begin{array}{l}\text { Clase } \\
\text { Coop } \\
\text { BCG } \\
2017\end{array}$ & \begin{tabular}{|c|} 
Cifra de \\
Ventas \\
2018 \\
(M/E) \\
\end{tabular} & \begin{tabular}{|c|}
$\%$ Cuota \\
Relativa \\
TOP10 \\
2018 \\
\end{tabular} & \begin{tabular}{|c|}
$\%$ Cuota \\
Crecimien \\
to 2017 a \\
2018
\end{tabular} & \begin{tabular}{|l} 
Clase \\
Coop \\
BCG \\
2018 \\
\end{tabular} & Actvidades cooperativizadas \\
\hline 1 & DCOOP & ANDALUCIAA & 2 & 208 & 468 & 937 & 1.005 & $18,28 \%$ & $7,28 \%$ & ES & 1.080 & $17,85 \%$ & $7,44 \%$ & VL & 1.230 & $18,69 \%$ & $13,89 \%$ & ES & $\begin{array}{l}\text { Aceite de oliva, Vino, Aceituna de } \\
\text { mesa, Suministros, Servicios y } \\
\text { Exportación. }\end{array}$ \\
\hline 2 & COREN & GALICIA & 2 & 10 & 3.240 & 950 & 1.000 & $18,19 \%$ & $5,26 \%$ & ES & 1.033 & $17,07 \%$ & $3,30 \%$ & VL & 1.078 & $16,38 \%$ & $4,36 \%$ & VL & \begin{tabular}{|l} 
Piensos, Avivolal, Vacuno de carne, \\
Porcino, Quesos, Leche, Servicios y \\
Exportación.
\end{tabular} \\
\hline 3 & GRUPOAN & NAVARRA & 2 & 160 & 1.681 & 716 & 767 & $13,95 \%$ & $7,12 \%$ & ES & 803 & $13,27 \%$ & $4,69 \%$ & VL & 1.074 & $16,32 \%$ & $33,75 \%$ & ES & $\begin{array}{l}\text { Cul. herbáceoss, Avicola, Hortalizas, } \\
\text { Carburantes, Inpust, Frutas, Porcino, } \\
\text { Piensos, Exportacion, Prod. Ecológ. }\end{array}$ \\
\hline 4 & $\begin{array}{l}\text { SAT CENTRAL } \\
\text { LECHERA } \\
\text { ASTURIANA }\end{array}$ & \begin{tabular}{|l|} 
PRINCIPADO \\
DE ASTURIAS
\end{tabular} & 1 & 7.470 & 14 & 708 & 673 & $12,24 \%$ & $-4,94 \%$ & VL & 746 & $12,33 \%$ & $10,85 \%$ & VL & 761 & $11,56 \%$ & $2,01 \%$ & VL & Leche de vaca y Servicios. \\
\hline 5 & ANECOOP & $\begin{array}{l}\text { COMUNIDAD } \\
\text { VALENCIANA }\end{array}$ & 2 & 68 & 236 & 614 & 663 & $12,06 \%$ & $7,98 \%$ & ES & 702 & $11,60 \%$ & $5,88 \%$ & VL & 760 & $11,55 \%$ & $8,26 \%$ & ES & $\begin{array}{l}\text { Ciricicos, Frutas, Hortalizas, Vino, } \\
\text { Exportación y Productis ecológicos. }\end{array}$ \\
\hline 6 & COVAP & ANDALUCIA & 1 & 7.301 & 707 & 415 & 405 & $7,37 \%$ & $-2,41 \%$ & PE & 441 & $7,29 \%$ & $8,89 \%$ & PE & 553 & $8,40 \%$ & $25,40 \%$ & $? ?$ & $\begin{array}{l}\text { Piensos, Vacuno de carne, Ovino de } \\
\text { carne, Porcino, Leche, Suministros, } \\
\text { Serv, Sección crédito y Exportación. }\end{array}$ \\
\hline 7 & COBADU & $\begin{array}{l}\text { CASTLLLAy } \\
\text { LEÓN }\end{array}$ & 1 & 9.753 & 182 & 275 & 272 & $4,95 \%$ & $-1,09 \%$ & PE & 309 & $5,11 \%$ & $13,60 \%$ & PE & 335 & $5,09 \%$ & $8,41 \%$ & $? ?$ & $\begin{array}{l}\text { Vacuno de carne, Ovivo de leche, } \\
\text { Porcino, Leche, Quesos, Suministos } \\
\text { Servicios v Tiendas. }\end{array}$ \\
\hline 8 & UNICA GROUP & ANDALUCIA & 2 & 22 & 37 & 230 & 260 & $4,73 \%$ & $13,04 \%$ & $? ?$ & 310 & $5,12 \%$ & $19,23 \%$ & PE & 282 & $4,28 \%$ & $-9,03 \%$ & PE & Hortalizas y Tiendas \\
\hline 9 & $\begin{array}{l}\text { AGRO } \\
\text { SEVILLA } \\
\text { ACEITUNAS }\end{array}$ & ANDALUCIA & 2 & 12 & 313 & 172 & 130 & $2,36 \%$ & $-24,71 \%$ & PE & 307 & $5,07 \%$ & $\mid 137,07 \%$ & $? ?$ & 258 & $3,92 \%$ & $-15,96 \%$ & PE & Aceitunas de mesa, Aceite de olva. \\
\hline 10 & ACOR & $\begin{array}{l}\text { CASTLLLAy } \\
\text { LEÓN }\end{array}$ & 1 & 4.470 & 554 & 312 & 323 & $5,88 \%$ & $3,53 \%$ & $? ?$ & 320 & $5,29 \%$ & $-0,93 \%$ & $\mathrm{PE}$ & 251 & $3,81 \%$ & $-21,60 \%$ & PE & $\begin{array}{l}\text { Azúcar, Cultivos herbáceos y } \\
\text { Suministros. }\end{array}$ \\
\hline & & & & & Totales . & 5.329 & 5.4981 & $100,00 \%$ & $11,06 \%$ & & 6.051 & $100 \%$ & $210,02 \%$ & & 6.582 & $100 \%$ & $49,48 \%$ & & \\
\hline
\end{tabular}

Fuente: OSCAE $(2018,2019,2020)$ y elaboración propia

Gráfica 4. Matriz BCG del TOP10 Cooperativas Agroalimentarias españolas, años 2016, 2017, 2018

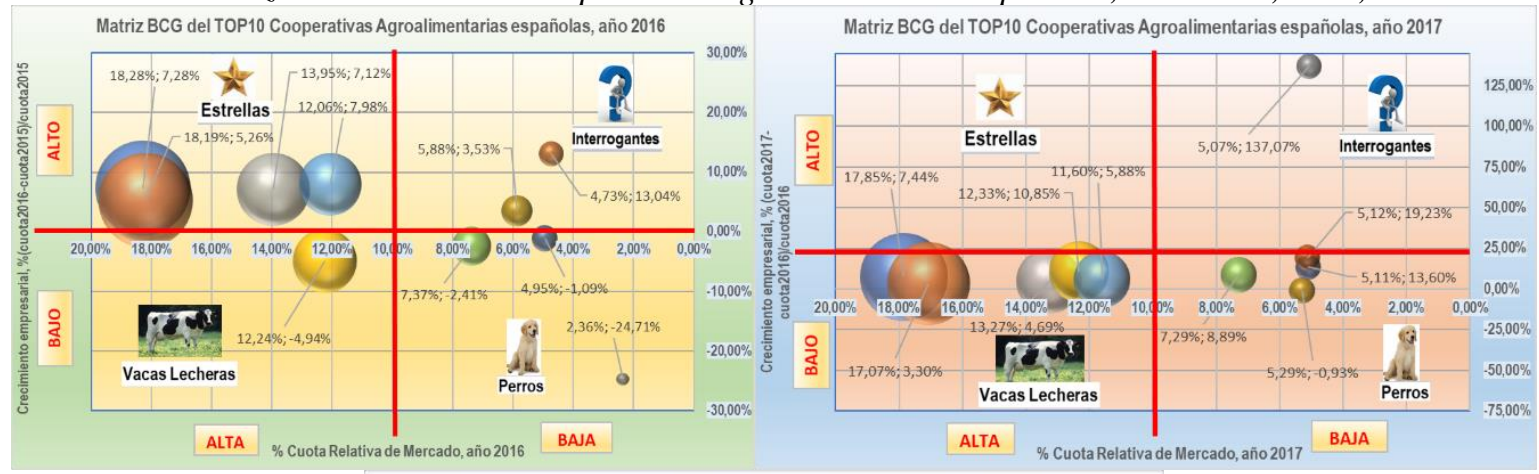

Matriz BCG del TOP10 Cooperativas Agroalimentarias españolas, año 2018

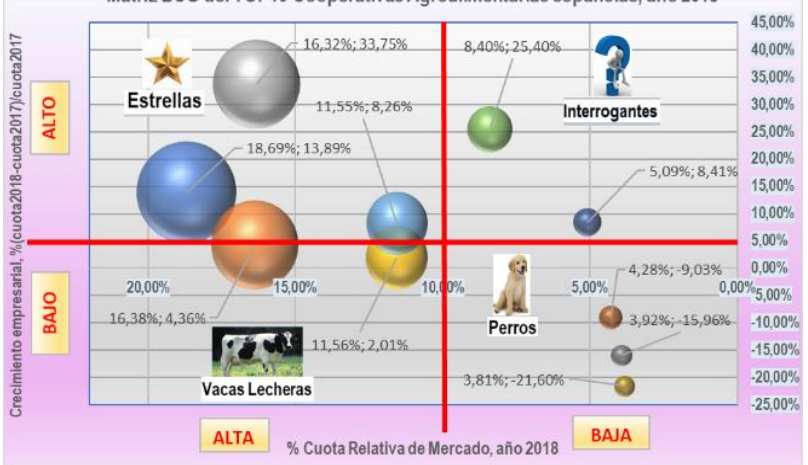

Fuente: OSCAE $(2018,2019,2020)$ y elaboración propia 
Un primer análisis de las TOP10 cooperativas agroalimentarias españolas nos indica que en 2016 un grupo de 5 cooperativas superaron el promedio del $10 \%$ de cuota relativa de mercado: DCOOP, COREN, GRUPO AN y ANECOOP como Estrellas; y la SAT CENTRAL LECHERA ASTURIANA como Vaca Lechera. Pasando a la siguiente campaña, en 2017 no se comportó como Estrella ninguna cooperativa; y como Vacas Lecheras se comportaron: DCOOP, COREN, GRUPO AN, SAT CENTRAL LECHERA ASTURIANA y ANECOOP, es decir fueron también 5 cooperativas que sobrepasaron el promedio del 10\% de cuota relativa de mercado. Finalmente, en 2018 fueron también 5 cooperativas las que franquearon el promedio del 10\% de cuota relativa de mercado: DCOOP, GRUPO AN y ANECOOP como Estrellas; y COREN y la SAT CENTRAL LECHERA ASTURIANA como Vacas Lecheras.

En los años 2016, 2017 y 2018 un total de 5 cooperativas presentaron un \% de la cuota relativa de mercado inferior al promedio del 10\%. En 2016, había 2 cooperativas Interrogantes (\% de la tasa de crecimiento del mercado por encima del promedio) y 3 cooperativas Perros (\% de la tasa de crecimiento del mercado por debajo del promedio); en 2017 hubo una sola cooperativa Interrogantes y 4 cooperativas Perros; y en 2018 había 2 cooperativas Interrogantes y 3 cooperativas Perro como en 2016.

Desde la campaña de 2015 a la de 2016 (ver Tabla 5), 6 cooperativas tuvieron un \% de tasa de crecimiento positivo en la cuota de mercado y otras 4 cooperativas restantes la tuvieron negativa, siendo el promedio del $\%$ de la tasa de crecimiento de $+1,11 \%$; desde 2016 a 2017, una gran mayoría de 9 cooperativas mostraron tasa de crecimiento positiva de la cuota de mercado y solo 1 presentó crecimiento negativo, siendo el promedio del $\%$ de tasa de crecimiento de $+21,00 \%$; finalmente, desde 2017 a 2018, se observaron 7 cooperativas que tuvieron crecimiento positivo de la cuota de mercado y 3 lo tuvieron negativo, siendo el promedio del $\%$ de tasa de crecimiento de $+4,95 \%$.

\section{CONCLUSIONES}

El panorama cooperativo agroalimentario español muestra un conjunto de Sociedades Cooperativas líderes, que aportan una componente importante a la actividad de producción primaria y transformación agroalimentaria en España.

Aunque restan bastantes asignaturas por cursar y aprobar en el mundo cooperativo agroalimentario español, con imperativos a seguir en acción para superar diversas problemáticas (de dimensión, de nivel de profesionalidad en la gestión y control, de equidad de género, de productividad del trabajo y de otros inputs relevantes, de poder de negociación ante retos comerciales globales, de financiación e ingeniería financiera, de innovación tecnológica, de proceso, de producto, de organización y de marketing, etc.), el itinerario seguido por el cooperativismo agroalimentario español, en general se cree que está girando hacia la buena dirección por parte del conjunto de empresas cooperativas líderes, que se esfuerzan en superar sus limitaciones y retos, y de esta forma integran y crecen, y adaptan su modelo organizativo para ir mejorando en su desempeño productivo, comercial, social y en relación al respeto medioambiental.

\section{BIBLIOGRAFÍA}

Colom A., Florensa R. (Directores) (2021). Cooperativismo Agroalimentario y las OPFH. Una panorámica mundial y la realidad actual de la Unión Europea y española. Libro colectivo como proyecto del INDEST-Universidad de Lleida. Ed. Thomson-Reuters-Aranzadi. 294 pág.

Colom A., Cos P., Florensa R. (2019). "Cooperativismo agroalimentario en Europa. Dimensión, gobernanza y análisis BCG de las sociedades cooperativas TOP25 de la UE-28 y TOP10 en España”. REVESCO, Revista de Estudios Cooperativos no 130, 1er Cuatrimestre 2019 (ISSN: 1885-8031).

Cooperativas Agro-alimentarias de España (2015). 25 años de cooperativismo agroalimentario democrático y unificado. Cajamar C. R. 2015, 420 pág.

OSCAE (2018). El Cooperativismo agroalimentario español. Informe Socioeconómico 2016. Observatorio Socioeconómico del Cooperativismo Agroalimentario Español, OSCAE-2017. Cooperativas Agroalimentarias de España-Ministerio de Agricultura, Pesca y Alimentación. Madrid.

OSCAE (2019). El Cooperativismo agroalimentario español. Informe Socioeconómico 2017. Observatorio Socioeconómico del Cooperativismo Agroalimentario Español, OSCAE-2018. Cooperativas Agroalimentarias de España-Ministerio de Agricultura, Pesca y Alimentación. Madrid.

OSCAE (2020). El Cooperativismo Agroalimentario Español. Informe Socioeconómico 2018. Observatorio Socioeconómico del Cooperativismo Agroalimentario Español, OSCAE-2019. Cooperativas Agroalimentarias de España-Ministerio de Agricultura, Pesca y Alimentación. Madrid. 